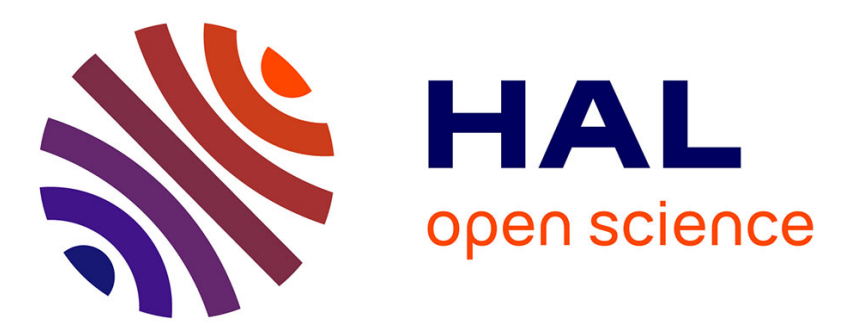

\title{
A micromechanical analysis of a local failure criterion for particle-reinforced composites
}

Johann Guilleminot, Djimedo Kondo, Christophe Binetruy, Stephane Panier, Said Hariri, Patricia Krawczak

\section{- To cite this version:}

Johann Guilleminot, Djimedo Kondo, Christophe Binetruy, Stephane Panier, Said Hariri, et al.. A micromechanical analysis of a local failure criterion for particle-reinforced composites. Composites Science and Technology, 2007, 67 (11-12), pp.2384. 10.1016/j.compscitech.2007.01.002 . hal-00498981

\section{HAL Id: hal-00498981 https://hal.science/hal-00498981}

Submitted on 9 Jul 2010

HAL is a multi-disciplinary open access archive for the deposit and dissemination of scientific research documents, whether they are published or not. The documents may come from teaching and research institutions in France or abroad, or from public or private research centers.
L'archive ouverte pluridisciplinaire HAL, est destinée au dépôt et à la diffusion de documents scientifiques de niveau recherche, publiés ou non, émanant des établissements d'enseignement et de recherche français ou étrangers, des laboratoires publics ou privés. 


\section{Accepted Manuscript}

A micromechanical analysis of a local failure criterion for particle-reinforced composites

Johann Guilleminot, Djimedo Kondo, Christophe Binetruy, Stephane Panier, Said Hariri, Patricia Krawczak

PII: S0266-3538(07)00038-3

DOI: 10.1016/j.compscitech.2007.01.002

Reference: CSTE 3571

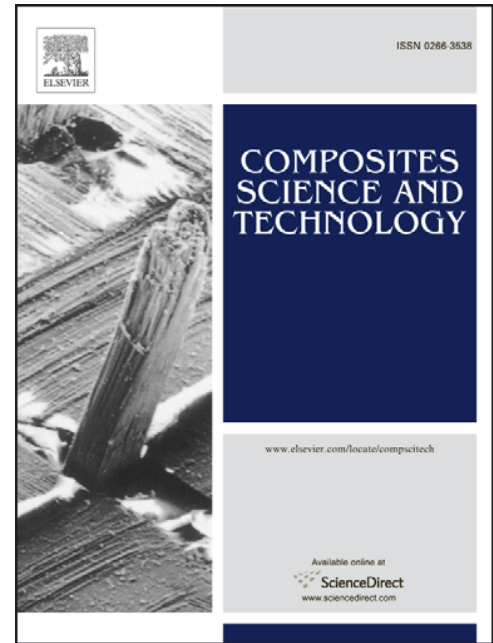

To appear in:

Composites Science and Technology

Received Date:

6 June 2006

Accepted Date:

12 January 2007

Please cite this article as: J. Guilleminot, D. Kondo, C. Binetruy, S. Panier, S. Hariri, P. Krawczak, A micromechanical analysis of a local failure criterion for particle-reinforced composites, Composites Science and Technology (2007), doi: 10.1016/j.compscitech.2007.01.002

This is a PDF file of an unedited manuscript that has been accepted for publication. As a service to our customers we are providing this early version of the manuscript. The manuscript will undergo copyediting, typesetting, and review of the resulting proof before it is published in its final form. Please note that during the production process errors may be discovered which could affect the content, and all legal disclaimers that apply to the journal pertain. 


\title{
A micromechanical analysis of a local failure criterion for particle-reinforced composites
}

\author{
Johann Guilleminot ${ }^{\mathrm{a}}$, Djimedo Kondo ${ }^{\mathrm{b}}$, Christophe Binetruy ${ }^{\mathrm{a}}$, \\ Stephane Panier ${ }^{a}$, Said Hariri ${ }^{a}$, Patricia Krawczak ${ }^{a}$ \\ ${ }^{a}$ Ecole des Mines de Douai, Polymers and Composites Technology 83 Mechanical \\ Engineering Department, 941 rue Charles Bourseul, BP 10838, 59508 Douai \\ Cedex, France \\ ${ }^{\mathrm{b}}$ Laboratoire de Mécanique de Lille-UMR CNRS 810\%, Université de Sciences et \\ Technologies Lille,
}

Cité Scientifique, Bd. Paul Langevin, 59655 Villeneuve d'Ascq Cedex, France

\begin{abstract}
The present paper aims at predicting the ultimate mechanical properties of a particlereinforced polymer using a micromechanical approach of a local failure criterion. The considered criterion includes both normal and shear stresses at the interface between the polymer matrix and the reinforcing material. In the case of rigid particles, a new closed-form expression of the interfacial stress concentration tensor is provided and simple analytical formula are proposed and compared for different homogenization schemes. A combination of these results with acoustic emission data allows the identification of the parameters involved in the local failure criterion. It has been shown that the predicted interfacial strength highly depends on the choice of a suitable homogenization scheme.
\end{abstract}

Key words: Particle-reinforced composites, Interfacial strength, Mechanical properties, Acoustic emission, Stress concentrations

\section{Introduction}

Polymer composites have been used for many years in several applications such as aircraft, space and marine structures, mass transport systems, automotive

Email addresses: guilleminot@ensm-douai.fr (Johann Guilleminot), kondo@univ-lille1.fr (Djimedo Kondo). 
industries, building and construction. The experience gained in all these applications showed that the in-service behavior of a polymer composite highly depends on the properties of the reinforcing particle-matrix or endless fibrematrix interface that contributes to stress transfer. The reinforcing particle can be a filler or a chopped fiber. This explains why the study of interfaces received much attention in order to understand phenomena and mechanisms involved there. Adhesion is one the most fundamental aspect addressed in this area because interfacial debonding is, with particle breakage, one of the basic local failure modes in particle-reinforced composites. The study of such local failure mechanism classically requires the use of a stress-based local approach. A suitable analysis can be performed by using micromechanical approaches which allow to relate the microscopic (local) stress field to the macroscopic stress tensor.The determination of the local stress field, particularly at the particle-matrix interface, depends on both the material microstructure and the choice of a suitable homogenization scheme. In this paper, we investigate the local criterion considered by Fitoussi et al. [2] and derive a new explicit formulation in the case of rigid particles, different homogenization schemes being considered and compared for this purpose. Moreover, the closed-form results are combined with acoustic emission data in order to evaluate the mechanical parameters involved in the criterion.

\section{Local debonding criterion and micromechanical analysis}

\subsection{Local failure criterion}

Let us consider a local failure criterion based on the stress field at the interface between the matrix and the reinforcing material. As previously used by Fitoussi et al. [2], an example of this type of criterion consists in a linear combination of the local normal and shear stresses and is defined as follows:

$$
\sigma_{n}^{\text {out }}+\beta \tau_{n}^{\text {out }}=R_{\text {int }}
$$

where $\sigma_{n}^{\text {out }}$ and $\tau_{n}^{\text {out }}$ are the interfacial normal and shear stresses respectively; $\beta$ and $R_{\text {int }}$ are two parameters to be identified by an iterative regression between both experimental and simulated results (see [2]). The local normal and shear stresses are classically defined as:

$$
\left\{\begin{array}{l}
\sigma_{n}^{\text {out }}=\underline{n}^{T} \cdot \boldsymbol{\sigma}^{\text {out }} \cdot \underline{n} \\
\tau_{n}^{\text {out }}=\sqrt{\left\|\boldsymbol{\sigma}^{\text {out }} \cdot \underline{n}\right\|^{2}-\left(\underline{n}^{T} \cdot \boldsymbol{\sigma}^{\text {out }} \cdot \underline{n}\right)^{2}}
\end{array}\right.
$$

where $\boldsymbol{\sigma}^{\text {out }}$ is the interfacial stress field and $\underline{n}$ is the outward unit normal to the boundary on the interface point under consideration. 


\subsection{Micromechanical formulation}

Requiring the displacement and the interfacial traction accross the boundary to be continuous, the components of the stress tensor just outside the inhomogeneity are found to be (see for instance [1]):

$$
\sigma_{i j}^{\text {out }}=\frac{1}{D(\underline{n})} \mathbb{C}_{i j k l}^{1}\left[\mathbb{S}_{k l a b}^{2}+\mathbb{N}_{k p}(\underline{n}) n_{l} n_{q} \Delta \mathbb{C}_{p q m n} \mathbb{S}_{m n a b}^{2}\right] \sigma_{a b}^{2 i n}
$$

where $\boldsymbol{\sigma}^{\text {out }}$ is the stress tensor at the interface, $\mathbb{C}^{1}$ is the stiffness tensor of the matrix; $\mathbb{C}^{2}$ and $\mathbb{S}^{2}$ are the stiffness and compliance tensors of the inhomogeneity respectively. $\Delta \mathbb{C}$ is defined by $\Delta \mathbb{C}=\mathbb{C}^{2}-\mathbb{C}^{1}, \sigma^{2}$ in is the stress tensor inside the inhomogeneity, $\mathbb{N}_{k p}$ and $D(\underline{n})$ are the cofactor and determinant, respectively, of the acoustic tensor $\mathbb{K}$ associated with $\mathbb{C}^{1}$ and the direction $\underline{n}$ :

$$
\begin{array}{r}
\mathbb{K}_{i k}=\mathbb{C}_{i j k l}^{1} n_{j} n_{l} \\
D(\underline{n})=\epsilon_{m n l} \mathbb{K}_{m 1} \mathbb{K}_{n 2} \mathbb{K}_{l 3} \\
\mathbb{N}_{i j}=\frac{1}{2} \in_{i k l} \in \in_{j m n} \mathbb{K}_{k m} \mathbb{K}_{l n}
\end{array}
$$

where $\epsilon_{i j k}$ are the components of the permutation tensor defined by:

$$
\epsilon_{i j k}=\left\{\begin{array}{l}
-1 \text { for the even permutation of } 1,2,3 \\
1 \text { for the odd permutation of } 1,2,3 \\
0 \text { for other cases }
\end{array}\right.
$$

A starting point of the micromechanical analysis consists in relating the microscopic and macroscopic stresses. Due to the linearity of this homogenization problem, the stress tensor $\boldsymbol{\sigma}^{2 \text { in }}$ inside the inhomogeneity is given by the stress concentration relation:

$$
\boldsymbol{\sigma}^{2 \text { in }}=\mathbb{B}^{2}: \Sigma
$$

where $\mathbb{B}^{2}$ is the stress concentration tensor for the inhomogeneity embedded in the solid matrix and $\boldsymbol{\Sigma}$ is the macroscopic stress tensor prescribed at the boundary of the representative elementary volume.

\subsection{Case of a matrix reinforced by infinitely rigid inclusions}

In order to derive closed-form expressions, we consider the case of rigid reinforcements (i.e. $\mathbb{S}^{2} \rightarrow 0$ ); we then rewritte the term $\Delta \mathbb{C}_{p q m n} \mathbb{S}_{m n a b}^{2}$ as:

$$
\Delta \mathbb{C}_{p q m n} \mathbb{S}_{m n a b}^{2}=\left(\mathbb{C}^{2}-\mathbb{C}^{1}\right)_{p q m n} \mathbb{S}_{m n a b}^{2}=\mathbb{I}_{p q a b}-\left(\mathbb{C}^{1}: \mathbb{S}^{2}\right)_{p q a b} \approx \mathbb{I}_{p q a b}
$$


where $\mathbb{I}$ is the symmetric fourth-order identity tensor defined by $\mathbb{I}_{\text {pqab }}=$ $\frac{1}{2}\left(\delta_{p a} \delta_{q b}+\delta_{p b} \delta_{q a}\right), \delta$ is the Kronecker delta $\left(\delta_{i j}=1\right.$ for $i=j$ and $\delta_{i j}=0$ for $i \neq j$ ). Taking into account (9), we have:

$$
\mathbb{N}_{k p}(\underline{n}) n_{l} n_{q} \Delta \mathbb{C}_{p q m n} \mathbb{S}_{m n a b}^{2}=\mathbb{N}_{k p}(\underline{n}) n_{l} n_{q} \mathbb{I}_{p q a b}
$$

which also reads

$$
\mathbb{N}_{k p}(\underline{n}) n_{l} n_{q} \Delta \mathbb{C}_{p q m n} \mathbb{S}_{m n a b}^{2}=\frac{1}{2}\left\{\mathbb{N}_{k a}(\underline{n}) n_{l} n_{b}+\mathbb{N}_{k b}(\underline{n}) n_{l} n_{a}\right\}=\mathbb{N}(\underline{n}) \underline{\bar{\otimes}}(\underline{n} \otimes \underline{n})
$$

where $(A \underline{\bar{\otimes}} B)_{k l a b}=\frac{1}{2}\left[A_{k a} B_{l b}+A_{k b} B_{l a}\right]$ for any second order tensors $\boldsymbol{A}$ and $\boldsymbol{B}$.

Then, equation (3) can be rewritten in a more readily and compact tensorial form:

$$
\boldsymbol{\sigma}^{\text {out }}=\frac{1}{D(\underline{n})} \mathbb{C}^{1}:[\mathbb{N}(\underline{n}) \underline{\bar{\otimes}}(\underline{n} \otimes \underline{n})]: \boldsymbol{\sigma}^{2 \text { in }}
$$

Let us recall now that for different homogenization schemes, the stress concentration tensor which enters (8) is classically given for the inhomogeneity by (see for instance [8]):

$$
\mathbb{B}^{2}=\mathbb{B}_{i}^{2}:\left(\sum_{k=1}^{2} f^{r} \mathbb{B}_{i}^{r}\right)^{-1}
$$

where $f^{r}$ is the volume fraction of the phase $r$ (the superscripts 0,1 and 2 denote some reference medium, the matrix and the inhomogeneity respectively) and $\mathbb{B}_{i}^{r}$ is defined by:

$$
\mathbb{B}_{i}^{r}=\left[\mathbb{I}+\mathbb{Q}^{0}:\left(\mathbb{S}^{r}-\mathbb{S}^{0}\right)\right]^{-1}
$$

where $\mathbb{S}^{r}$ is the compliance tensor of the phase $r$ and $\mathbb{Q}^{0}$ is defined by :

$$
\mathbb{Q}^{0}=\mathbb{C}^{0}-\mathbb{C}^{0}: \mathbb{P}_{0}^{2}: \mathbb{C}^{0}
$$

$\mathbb{P}_{0}^{2}$ is the Hill tensor (see [11]). Since $\mathbb{C}^{0}: \mathbb{S}^{0}=\mathbb{I}$ and $\mathbb{S}^{2} \rightarrow 0$, one easily gets:

$$
\left\{\begin{array}{l}
\mathbb{B}_{i}^{1}=\left[\mathbb{I}+\mathbb{Q}^{0}:\left(\mathbb{S}^{1}-\mathbb{S}^{0}\right)\right]^{-1} \\
\mathbb{B}_{i}^{2} \approx\left(\mathbb{C}^{0}: \mathbb{P}_{0}^{2}\right)^{-1}
\end{array}\right.
$$

Substituting (16) into (13) and recalling that $(\mathbb{A}: \mathbb{B})^{-1}=\mathbb{B}^{-1}: \mathbb{A}^{-1}(\mathbb{A}$ and $\mathbb{B}$ being any symmetric fourth-order tensor, i.e. $\left.\mathbb{A}_{i j k l}=\mathbb{A}_{j i k l}=\mathbb{A}_{i j l k}=\mathbb{A}_{k l i j}\right)$ 
and $f^{1}=1-f^{2}$, we obtain for the two phase composites:

$$
\begin{aligned}
& \mathbb{B}^{2}=\mathbb{B}_{i}^{2}:\left[\left(1-f^{2}\right) \mathbb{B}_{i}^{1}+f^{2} \mathbb{B}_{i}^{2}\right]^{-1} \approx\left[\left(1-f^{2}\right)\left[\mathbb{I}+\mathbb{Q}^{0}:\left(\mathbb{S}^{1}-\mathbb{S}^{0}\right)\right]^{-1}: \mathbb{C}^{0}: \mathbb{P}_{0}^{2}+\right. \\
& \left.f^{2} \mathbb{I}\right]^{-1}
\end{aligned}
$$

and then, we finally define a new tensor,

$$
\begin{aligned}
& \mathbb{B}^{\text {out }}=\frac{1}{D(\underline{n})} \mathbb{C}^{1}:[\mathbb{N}(\underline{n}) \underline{\bar{\otimes}}(\underline{n} \otimes \underline{n})]:\left[\left(1-f^{2}\right)\left[\mathbb{I}+\mathbb{Q}^{0}:\left(\mathbb{S}^{1}-\mathbb{S}^{0}\right)\right]^{-1}: \mathbb{C}^{0}: \mathbb{P}_{0}^{2}+\right. \\
& \left.f^{2} \mathbb{I}\right]^{-1}
\end{aligned}
$$

such as:

$$
\boldsymbol{\sigma}^{\text {out }}=\mathbb{B}^{\text {out }}: \Sigma
$$

$\mathbb{B}^{\text {out }}$ can be considered, for rigid inhomogeneities, as a concentration tensor for the interfacial stress field: it is readily seen that this new tensor $\mathbb{B}^{\text {out }}$ depends on both the geometry of the inhomogeneity (via the tensor $\mathbb{P}_{0}^{2}$ ) and the properties of the reference medium denoted by the superscript 0 . Therefore, note that the expression of $\mathbb{B}^{\text {out }}$ clearly depends on the homogenization scheme (see section 2.4).

\subsection{Role of the homogenization schemes}

Different homogenization schemes are going to be considered in the following. For the dilute estimate (also known as the Eshelby's solution, see for instance [4]), classically devoted to non interacting inhomogeneities (at dilute concentration), the matrix is considered as the reference medium (i.e. $\mathbb{S}^{0}=\mathbb{S}^{1}$ ) and is subjected to the macroscopic stress tensor. The validity of this approach corresponds to $f^{2} \rightarrow 0$ and thus to, $\mathbb{B}^{2} \rightarrow\left(\mathbb{C}^{1}: \mathbb{P}_{0}^{2}\right)^{-1}$. We recall that in general, $\mathbb{P}_{0}^{2}=\mathbb{S}_{E S H}:\left(\mathbb{C}^{0}\right)^{-1}$, where $\mathbb{S}_{E S H}$ is the Eshelby tensor which depends on both the geometry of the inhomogeneity and the reference medium (see [1]). Therefore, eqn (18) yields:

$$
\mathbb{B}_{E S H}^{\text {out }}=\frac{1}{D(\underline{n})} \mathbb{C}^{1}:[\mathbb{N}(\underline{n}) \underline{\bar{\otimes}}(\underline{n} \otimes \underline{n})]:\left(\mathbb{C}^{1}: \mathbb{P}_{1}^{2}\right)^{-1}
$$

where obviously, $\mathbb{P}_{1}^{2}=\left(\mathbb{P}_{0}^{2}\right)_{\text {Dilute estimate }}=\mathbb{S}_{E S H}:\left(\mathbb{C}^{1}\right)^{-1}$.

For the Mori-Tanaka estimate [10] (see also [5]), which allows to take into account the interactions between the inhomogeneities, the solid matrix is still considered as the reference medium, but is now subjected to its own stress. It 
follows that:

$$
\mathbb{B}_{M T}^{\text {out }}=\frac{1}{D(\underline{n})} \mathbb{C}^{1}:[\mathbb{N}(\underline{n}) \underline{\bar{\otimes}}(\underline{n} \otimes \underline{n})]:\left[\left(1-f^{2}\right) \mathbb{C}^{1}: \mathbb{P}_{1}^{2}+f^{2} \mathbb{I}\right]^{-1}
$$

In the Generalized Self-Consistent scheme [6], the reference medium is the unknown effective medium denoted by $S C$, that is $\mathbb{S}^{0}=\mathbb{S}^{S C}$. Then, eqn (18) becomes:

$$
\begin{aligned}
& \mathbb{B}_{S C}^{\text {out }}=\frac{1}{D(\underline{n})} \mathbb{C}^{1}:[\mathbb{N}(\underline{n}) \underline{\bar{\otimes}}(\underline{n} \otimes \underline{n})]:\left[\left(1-f^{2}\right)[\mathbb{I}+\right. \\
& \left.\left.\mathbb{Q}^{S C}:\left(\mathbb{S}^{1}-\mathbb{S}^{S C}\right)\right]^{-1}: \mathbb{C}^{S C}: \mathbb{P}_{S C}^{2}+f^{2} \mathbb{I}\right]^{-1}
\end{aligned}
$$

Finally, the Ponte-Castaneda and Willis estimate [7] aims at taking into account not only the interaction effect, but also the spatial distribution of the reinforcing material by means of a new tensor $\mathbb{P}_{d}$ (see [7] for a detailed presentation as well as the expression of $\mathbb{P}_{d}$ ). In this approach, the tensor $\mathbb{P}_{1}^{2}$ is then replaced by $\frac{1}{1-f^{2}}\left[\mathbb{P}_{1}^{2}-f^{2} \mathbb{P}_{d}\right]$ and we get:

$$
\mathbb{B}_{P C W}^{\text {out }}=\frac{1}{D(\underline{n})} \mathbb{C}^{1}:[\mathbb{N}(\underline{n}) \underline{\bar{\otimes}}(\underline{n} \otimes \underline{n})]:\left(\mathbb{C}^{1}:\left[\mathbb{P}_{1}^{2}-f^{2} \mathbb{P}_{d}\right]+f^{2} \mathbb{I}\right)^{-1}
$$

\section{Case of a polymer-matrix composite reinforced by spherical in- homogeneities: a comparison between micromechanical schemes}

\subsection{Introduction}

In this section, we consider an isotropic unsaturated polyester matrix reinforced by E-Glass spherical beads (volume fraction : $30 \%$ ). Our aim here is to determine the sensitivities of both the parameter $R_{\text {int }}$ and the local stress field to the homogenization scheme. The spherical particles have been burnt five hours at $520^{\circ} \mathrm{C}$ to remove sizing in order to promote interfacial debonding instead of matrix cracking. Fig. (1) shows a SEM inspection of the composite material. The lack of polymer matrix at the particle surface, i.e. the particle surface is smooth, gives the evidence that interfacial debonding is the damage mechanism.

The values of the elastic moduli and Poisson's ratios for each phase (the superscripts 1 and 2 correspond to the matrix and the inhomogeneity respectively) 


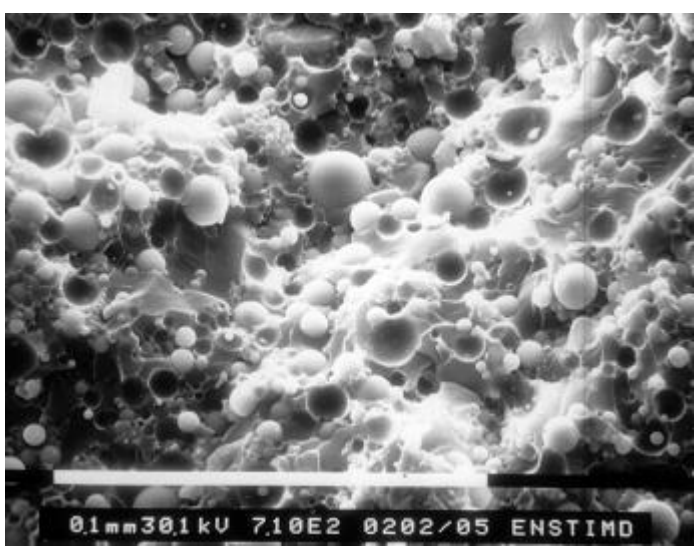

Figure 1. SEM micrography of the glass beads-filled unsaturated polyester resin are:

$$
\left\{\begin{array}{l}
E^{1}=2.7[G P a] \\
\nu^{1}=0.37 \\
E^{2}=73[G P a] \\
\nu^{2}=0.22
\end{array}\right.
$$

The consideration of these data has basically motivated the assumption of rigid particles.

For spherical inhomogeneities, the Hill tensor $\mathbb{P}_{1}^{2}$ is given by (see [11] or [1]):

$$
\mathbb{P}_{1}^{2}=\frac{\alpha^{1}}{3 k^{1}} \mathbb{J}+\frac{\beta^{1}}{2 \mu^{1}} \mathbb{K}
$$

with

$$
\alpha^{1}=\frac{3 k^{1}}{3 k^{1}+4 \mu^{1}} ; \quad \beta^{1}=\frac{6\left(k^{1}+2 \mu^{1}\right)}{5\left(3 k^{1}+4 \mu^{1}\right)}
$$

$k^{1}$ and $\mu^{1}$ are the bulk and shear moduli of the matrix respectively. $\mathbb{J}$ and $\mathbb{K}$ are the classical symmetric fourth-order tensors defined by $\mathbb{J}=\frac{1}{3} \boldsymbol{i} \otimes \boldsymbol{i}$ and $\mathbb{K}=\mathbb{I}-\mathbb{J}, \boldsymbol{i}$ is the second-order symmetric identity tensor $: i_{i j}=\delta_{i j}$. Due to the isotropy of the constitutive phases, the stiffness tensor for the phase $r$ can be written as:

$$
\mathbb{C}^{r}=3 k^{r} \mathbb{J}+2 \mu^{r} \mathbb{K}
$$

and then, the compliance tensor reads:

$$
\mathbb{S}^{r}=\left[\mathbb{C}^{r}\right]^{-1}=\frac{1}{3 k^{r}} \mathbb{J}+\frac{1}{2 \mu^{r}} \mathbb{K}
$$

In the case of the $S C$ scheme, the Hill tensor $\mathbb{P}_{S C}^{2}$ is given by:

$$
\mathbb{P}_{S C}^{2}=\mathbb{S}_{E S H}:\left(\mathbb{C}^{S C}\right)^{-1}=\frac{\alpha^{S C}}{3 k^{S C}} \mathbb{J}+\frac{\beta^{S C}}{2 \mu^{S C}} \mathbb{K}
$$


for which we recall that $k^{S C}$ and $\mu^{S C}$ are the effective bulk and shear moduli of the composite respectively. $\alpha^{S C}$ and $\beta^{S C}$ are obtained from (26) in which $k^{1}$ and $\mu^{1}$ must be replaced by $k^{S C}$ and $\mu^{S C}$ respectively. Moreover, experimental results in the case of a tensile test suggest that the debonding does appear at the pole of the spherical particle, as illustrated on Fig. (2).

Then, we note that:

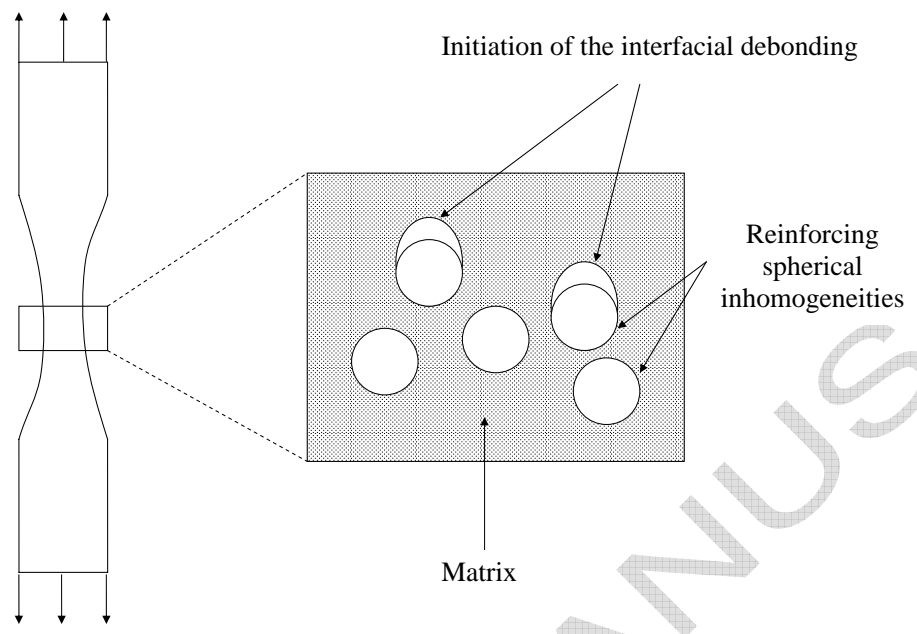

Figure 2. Interfacial debonding during a tensile test

$$
\sigma_{n, \text { pole }}^{\text {out }}=\operatorname{Max}\left(\sigma_{n}^{\text {out }}\right), \tau_{n, \text { pole }}^{\text {out }}=0
$$

and recalling eqn (1), we get:

$$
R_{\text {int }}=\sigma_{n, \text { pole }}^{\text {out }}
$$

It follows that $R_{\text {int }}$ can be determined by a pure macroscopic uniaxial tensile test coupled with an acoustic emission measure, as the debonding macroscopic force $F_{\text {deb }}$ (and so, the interfacial failure) can be determined by comparing the acoustic emission (AE) results (that are the counts and the amplitude - in $\mathrm{dB}$ - vs. time - in seconds -) with the tensile test results (the force - in N - vs. time); see section (3.3). We recall here that AE is widely used for non destructive control as well as to identify and to monitor damage mechanisms and evolution (see for instance [9] or [3]). In fact, each failure mode can be characterized by a range of amplitude; the debonding phenomena basically corresponds to the range [58...68] dB. Thus, we define the macroscopic tensile stress tensor $\Sigma$ (which is used for the micromechanical analysis) by :

$$
\Sigma=\operatorname{Diag}\left[0,0, \Sigma_{33}\right]
$$




\subsection{Sensitivity of the local stress field}

Due to the geometrical and material symmetries of the reinforcing inhomogeneities, the local normal and shear stresses are characterized by using a parametrization of the unit normal $\underline{n}$, that is (see Fig. (3)):

$$
\underline{n}=[\cos (\alpha), 0, \sin (\alpha)]^{T}, \alpha \in\left(0, \frac{\pi}{2}\right)
$$

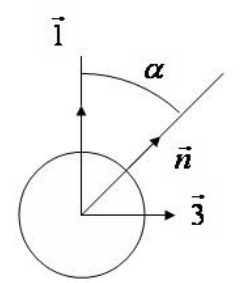

Figure 3. Parametrization of the unit normal

Without any loss of generality, we consider an unit macroscopic tensile stress tensor $\boldsymbol{\Sigma}=\operatorname{Diag}[0,0,1]$ and the local normal and shear stress fields are computed, for the different homogenization schemes, by using eqns (20), (21), (22) or (23) combined with eqn (19). The results are shown on Fig. (4) and (5).

The following remarks can be done:

- For both normal and shear stresses: the different homogenization schemes provide the same profiles but different amplitudes; moreover, we note that the local normal and shear stresses are not maxima at the same interfacial point, which means that the failure can occur at points located between the two points defining the maximum of the normal and shear stresses respectively. The biggest difference between the estimates is observed at the point of maximal stress, that is for instance $\alpha=\frac{\pi}{2}$ for the normal component.

- For the local normal stress: as expected, the component is minimal and null at the equator of the spherical inhomogeneity, while reaching its maximum at the pole. As $\alpha$ increases from 0 to $\frac{\pi}{2}$, the local normal stress starts to decrease from 0 to its minimal value, and thus defines a local compression; the component then starts increasing from its minimal to its maximal positive value obtained for $\alpha=\frac{\pi}{2}$ (change from a local compression to a local traction).

- For the local shear stress: the shear component is positive and null at both the equator and the pole of the reinforcing inhomogeneity.

The maximum relative errors $\epsilon[\%]$ between the estimates are also computed. Note that these errors for the local normal and shear stresses are exactly the same, as the use of different homogenization schemes basically results 


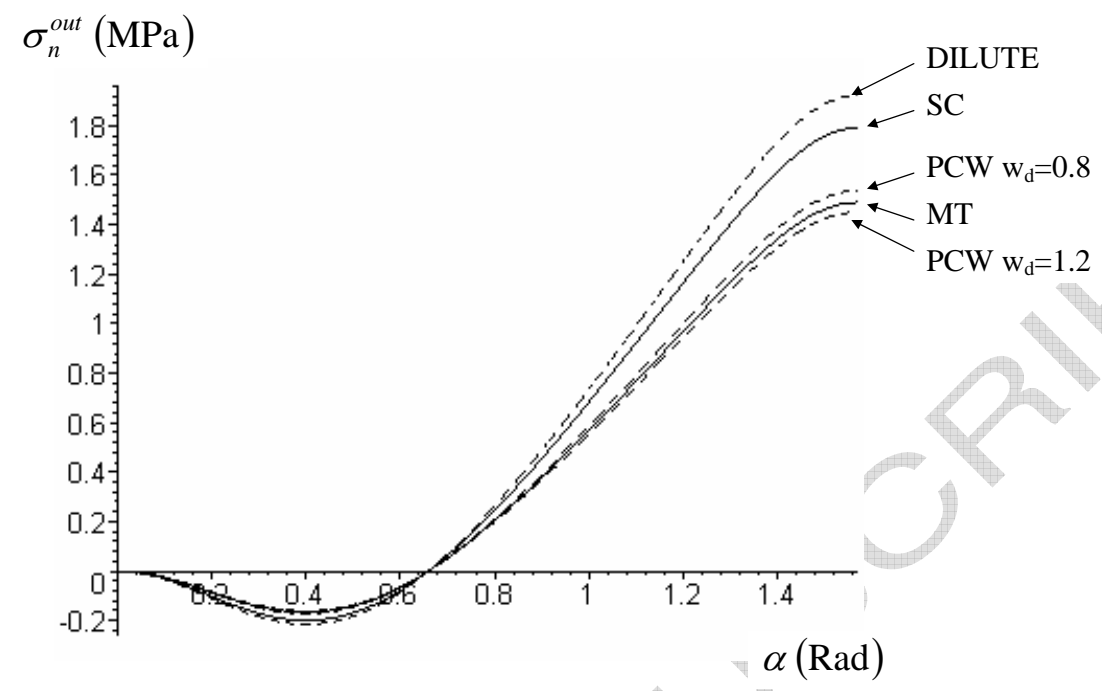

Figure 4. Distribution of the local normal stress

in different predictions for the interfacial stress field which is used for the determination of both local normal and shear stresses. The results are reported in table (1).

\begin{tabular}{|c|c|c|c|c|c|}
\hline & Dilute & Mori-Tanaka & SC & PCW $w_{d}=0.8$ & PCW $w_{d}=1.2$ \\
\hline Dilute & $0[\%]$ & $22.6[\%]$ & $6.9[\%]$ & $20.1[\%]$ & $24.6[\%]$ \\
\hline Mori-Tanaka & & $0[\%]$ & $16.8[\%]$ & $3.1[\%]$ & $2.6[\%]$ \\
\hline SC & & & $0[\%]$ & $14.2[\%]$ & $19[\%]$ \\
\hline PCW $w_{d}:=0.8$ & & & & $0[\%]$ & $5.6[\%]$ \\
\hline PCW $w_{d}:=1.2$ & & & & & $0[\%]$ \\
\hline
\end{tabular}

Table 1

Maximum relative errors for the different homogenization schemes

\subsection{Sensitivity of the parameter $R_{\text {int }}$}

For application purposes, the tensile test has been carried out on a Instron tensile machine and coupled with an acoustic emission system (threshold: 30 dB). Eight tensile tests were carried out (see Fig. (6)) and a mean value for 


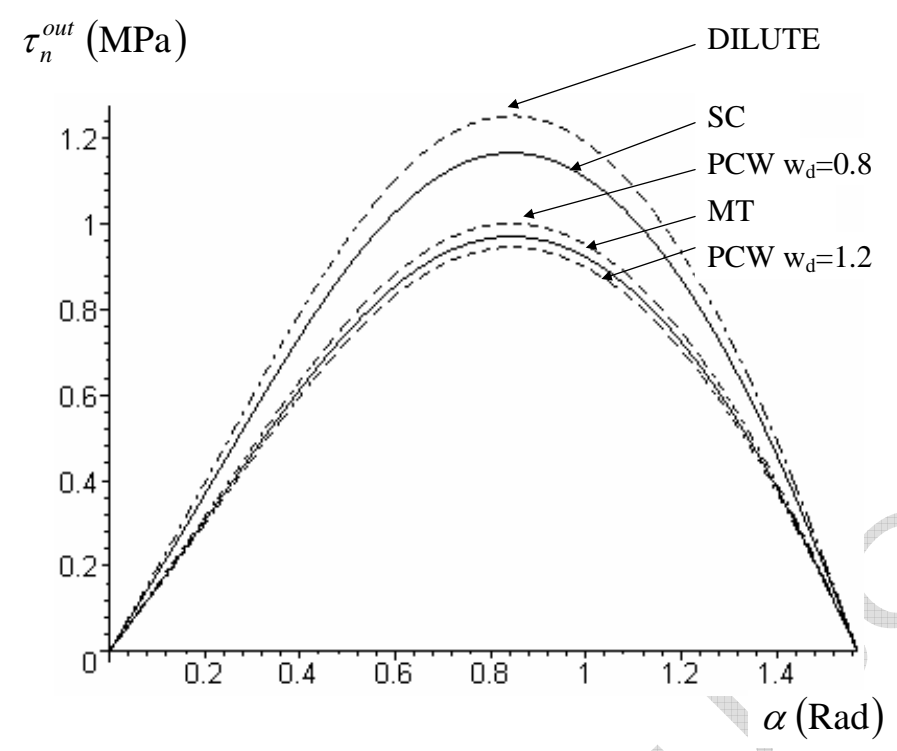

Figure 5. Distribution of the local shear stress

$F_{\text {deb }}$ was obtained:

$$
F_{\mathrm{deb}} \approx 39.42[d a N]
$$

Given a mean section of $S=37.84\left[\mathrm{~mm}^{2}\right]$, we find the uniaxial debonding macroscopic stress as:

$$
\Sigma_{\mathrm{deb}}=\frac{F_{\mathrm{deb}}}{S} \approx 10.42[M P a]
$$

Substituting $\Sigma_{33}=\Sigma_{\text {deb }}$ in eqns (20), (21), (22) and (23) and using eqn (19), we finally derive the estimates of $R_{\text {int }}$ for the different homogenization schemes under consideration (see table (2)).

Table 2

\begin{tabular}{|c|c|}
\hline Homogenization Scheme & Estimate of $R_{\text {int }}$ \\
\hline Dilute & $20.06[\mathrm{MPa}]$ \\
\hline Mori-Tanaka & $15.53[\mathrm{MPa}]$ \\
\hline $\mathrm{SC}$ & $18.67[\mathrm{MPa}]$ \\
\hline $\mathrm{PCW} w_{d}=0.8$ & $16.03[\mathrm{MPa}]$ \\
\hline $\mathrm{PCW} w_{d}=1.2$ & $15.13[\mathrm{MPa}]$ \\
\hline
\end{tabular}

Estimates of $R_{\text {int }}$ 


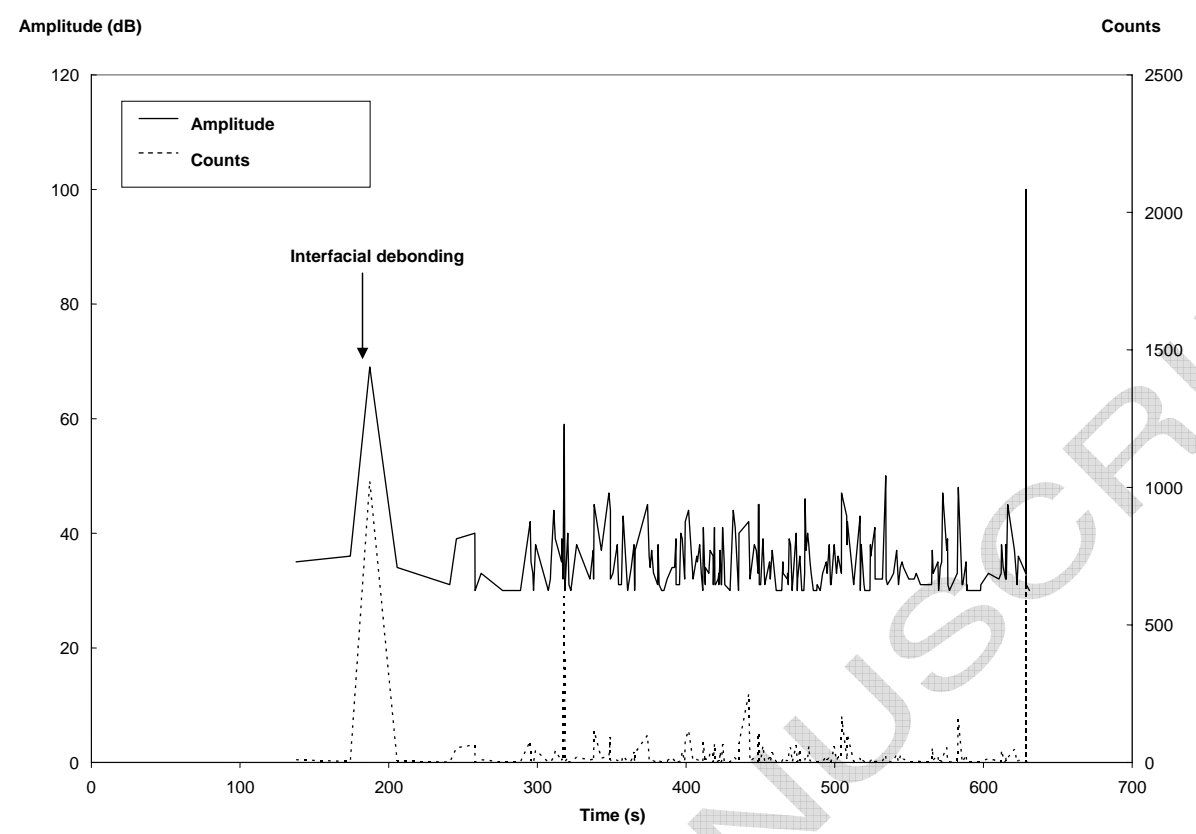

Figure 6. AE spectra (amplitudes and counts vs. time) used for the identification of the debonding macroscopic force

It is seen that the predictions for the mechanical parameter $R_{\text {int }}$ are very different depending on the micromechanical formulation under consideration: a maximum relative error of $24.6[\%]$ is for instance observed between the Ponte-Castaneda and Willis $\left(w_{d}=1.2\right)$ estimate and the Eshelby's solution which seems to overestimate the interfacial strength.

\section{Concluding remarks}

The role of the homogenization scheme in the analysis of a local failure criterion has been investigated. In particular, a new closed-form expression of the interfacial stress concentration tensor has been proposed in the case of rigid particle-reinforced composites. The proposed methodology can be easily applied to a more general class of materials (for instance thermoplastics reinforced with short or long fibres). The coupling of such a micromechanical analysis with acoustic emission data allowed us to determine the interfacial strength whose sensitivity to the homogenization scheme is emphasized. Thus, it is observed that the predicted value of the interfacial strength (and in gen- 
eral, of the effective mechanical properties) strongly depends on the homogenization scheme which in practice has to be carefully chosen according to the real morphology of the material.

\section{References}

[1] Mura, T., "Micromechanics of defects in solids", M. Nijhoff Publ., The Hague, The Netherlands, 1987.

[2] Fitoussi, J., Guo, G., Baptiste, D., "Determination of a tridimensional failure criterion at the fibre/matrix interface of an organic-matrix/discontinuousreinforcement composite", Composites Science and Technology, Vol. 56, p.755$760,1995$.

[3] Barre, S., Benzeggah, M. L., "On the use of acoustic emission to investigate damage mechanisms in glass-fibre-reinforced polypropylene", Composites Science and Technology, Vol. 54, p.369-376, 1994.

[4] Eshelby, J. D., "The determination of the elastic field of an ellipsoidal inclusion, and related problems", Proceedings of the Royal Society, Series A, Vol. 241, p.376-396, 1957.

[5] Benveniste, Y., "A new approach to the application of Mori-Tanaka's theory in composite materials", Mechanics of Materials, Vol. 6, p.147-157, 1987.

[6] Christensen, R. M., "A critical evaluation for a class of micromechanics models", J. Mech. Phys. Solids, Vol. 38, p.379-404, 1988.

[7] Ponte-Castaneda, P., Willis, J. R., "The effect of spatial distribution on the effective behavior of composite materials and cracked media", J. Mech. Phys. Solids, Vol. 43, p.1919-1951, 1995.

[8] Bornert, M., Bretheau, T., Gilormini, P., "Homogenization in Mechanics of Materials", Iste Publishing Company, 2006.

[9] Lariviere, D., Krawczak, P., Tiberi, C., Lucas, P., "Acoustic emission applied to failure analysis of commingled yarn $\mathrm{GF} / \mathrm{PP}$ composites in transverse tension and mode I delamination", Advanced Composites Letters, Vol. 12, No. 5, p.191202, 2003.

[10] Mori, T. and Tanaka, K. "Average stress in matrix and average elastic energy of materials with misfitting inclusions", Acta Metall. 21, 571-574, 1973.

[11] Nemat-Nasser, S., Hori, S., "Micromechanics: overall properties of heterogeneous materials", North-Holland series in applied mathematics and mechanics, The Netherlands, 1993. 